\title{
Hyperinsulinemia, glucose intolerance, and dyslipidemia induced by acute inhibition of phosphoinositide 3-kinase signaling in the liver
}

\author{
Kazuaki Miyake, Wataru Ogawa, Michihiro Matsumoto, Takehiro Nakamura, \\ Hiroshi Sakaue, and Masato Kasuga
}

Department of Clinical Molecular Medicine, Division of Diabetes, Digestive and Kidney Disease, Kobe University Graduate School of Medicine, Kobe, Japan

\begin{abstract}
The physiological relevance of phosphoinositide 3-kinase (PI 3-K) signaling in the liver to fuel homeostasis was investigated. Systemic infusion of an adenovirus encoding a dominant negative mutant of PI 3-K $(\Delta \mathrm{p} 85)$ resulted in liver-specific expression of this protein and in inhibition of the insulininduced activation of PI 3-K in the liver within 3 days, without affecting insulin signaling in skeletal muscle. Hepatic expression of $\Delta \mathrm{p} 85$ led to hyperinsulinemia and to a marked increase in blood glucose concentration in response to oral glucose intake. The increases in both glycogen and glucose 6-phosphate content, as well as in Akt and glycogen synthase activities in the liver, that were induced by glucose intake were markedly impaired in mice expressing $\Delta \mathrm{p} 85$. Despite an upregulation of mRNAs for gluconeogenic enzymes apparent in the liver of these animals, the fasting blood glucose concentration was increased only slightly, and the serum concentrations of gluconeogenic precursors were reduced. However, administration of pyruvate, a substrate for gluconeogenesis, resulted in an exaggerated increase in blood glucose concentration. In the fasted state, the mass of adipose tissue of the mice was about 1.5 times that in control mice. The mice also exhibited marked decreases in the serum concentrations of FFAs and triglyceride and suppression of insulin-induced PI 3-K activation in adipose tissue, probably due to the associated hyperinsulinemia. PI 3-K activity in the liver is thus essential for normal carbohydrate and lipid metabolism in living animals.
\end{abstract}

J. Clin. Invest. 110:1483-1491 (2002). doi:10.1172/JCI200215880.

\section{Introduction}

Among the various signaling molecules activated by insulin, a number of in vitro studies indicate that the class $\mathrm{I}_{\mathrm{A}}$ phosphoinositide 3-kinase (PI 3-K), which consists of a 110-kDa catalytic subunit and an Src homology 2 domain-containing regulatory subunit, plays a key role in the metabolic actions of this hormone $(1,2)$. Wortmannin and LY 294002, inhibitors of both class $I_{A}$ and class $I_{B}$ PI 3-Ks, thus prevent a variety of biological effects of insulin $(1,2)$. Ectopic expression of a dominant negative form of class $\mathrm{I}_{\mathrm{A}}$ PI 3-K has also revealed the importance of this enzyme in metabolic control by insulin (3-6). Furthermore, analysis of human subjects and animal models has indicated the existence of a

Received for publication May 8, 2002, and accepted in revised form October 1, 2002

Address correspondence to: Wataru Ogawa, Department of Clinical Molecular Medicine, Division of Diabetes and Digestive and Kidney Diseases, Kobe University Graduate School of Medicine, 7-5-1 Kusunoki-cho, Chuo-ku, Kobe 650-0017, Japan. Phone: 81-78-382-5861; Fax: 81-78-382-2080;

E-mail: ogawa@med.kobe-u.ac.jp.

Conflict of interest: The authors have declared that no conflict of interest exists.

Nonstandard abbreviations used: phosphoinositide 3-kinase (PI 3-K); insulin receptor substrate (IRS); insulin receptor (IR); glycogen synthase kinase (GSK); 3-mercaptopicolinic acid (3-MPA); phosphoenolpyruvate carboxykinase (PEPCK); sterol regulatory element-binding protein-1 (SREBP-1). correlation between impaired activation of PI 3-K in target tissues of insulin and insulin resistance in vivo (7-9). These various observations suggest that a disturbance of signaling mediated by this lipid kinase might contribute to the development of insulin resistance and, consequently, to the pathogenesis of type 2 diabetes.

Targeted gene disruption is also an important tool for investigating the role of specific proteins in vivo. Genes encoding three different isoforms ( $110 \alpha, \mathrm{p} 110 \beta$, and $\mathrm{p} 110 \gamma$ ) of the catalytic subunit of class $\mathrm{I}_{\mathrm{A}}$ PI $3-\mathrm{K}$ have been identified (2). Disruption of the p110 $\alpha$ gene, which is widely expressed among tissues, resulted in embryonic death in mice (10). Three genes encoding regulatory subunits ( $\mathrm{p} 85 \alpha, \mathrm{p} 85 \beta$, and $\mathrm{p} 55 \gamma$ ) of class $\mathrm{I}_{\mathrm{A}}$ PI 3-K have been identified, and that for $\mathrm{p} 85 \alpha$ also gives rise to two smaller variant proteins known as $\mathrm{p} 55 \alpha$ and p50 $\alpha$ (or AS53) (2). The metabolic phenotypes of mice lacking the $p 85 \alpha$ gene $(11,12)$ or the $p 85 \beta$ gene $(13)$, as well as of those selectively deficient in the $\mathrm{p} 85 \alpha$ protein (14), have been described; unexpectedly, all these animals exhibited lower blood glucose concentrations and increased insulin sensitivity compared with controls. Although the mechanism responsible for these phenotypes remains unclear, the insulin-induced association of PI 3-K activity with tyrosine-phosphorylated proteins or with insulin receptor substrate (IRS) proteins, shown to be important for insulin action by in vitro studies $(1$, $2)$, was only partially $(11,14)$ or minimally $(12,13)$ 
attenuated in the targeted mice. The metabolic impact of prevention of the insulin-dependent activation of PI $3-\mathrm{K}$ in vivo has thus not been elucidated to date.

Given that both humans with diabetes and many animal models of insulin resistance manifest defects in insulin responses in multiple tissues, it has been difficult to determine which impairment in which tissue is primarily responsible for a particular metabolic disturbance. Although several mouse models have been established that lack a specific protein in a specific tissue, these models have still exhibited abnormalities in multiple organs, probably due to secondary effects of chronic metabolic alterations $(15,16)$.

We have now investigated the metabolic impact of inhibition of hepatic PI 3-K signaling in vivo by systemic infusion of an adenoviral vector encoding a dominant negative mutant of class $\mathrm{I}_{\mathrm{A}}$ PI $3-\mathrm{K}$ in mice. This procedure resulted in liver-specific expression of the mutant protein and in almost complete inhibition of the insulininduced increase in hepatic PI 3-K activity within 3 days, a time course consistent with minimization of secondary effects due to chronic metabolic alterations. The acute inhibition of hepatic PI 3-K signaling resulted in several abnormalities in glucose and lipid metabolism.

\section{Methods}

Animals, adenoviruses, and statistical analysis. An adenoviral vector $(A x C A \Delta p 85)$ encoding a dominant negative mutant of class $\mathrm{I}_{\mathrm{A}}$ PI $3-\mathrm{K}$, consisting of bovine $\mathrm{p} 85 \alpha$ lacking the binding site for the catalytic subunit, was described previously (3), and an adenovirus encoding $\beta$-galactosidase (AxCALacZ) was kindly provided by I. Saito (University of Tokyo, Tokyo, Japan). Male BALB/c mice (8-10 weeks of age) were injected, via the tail vein, with adenoviral vectors at a dose of $5 \times 10^{8}$ plaqueforming units (dissolved in $150 \mu$ l of saline) per animal or with $150 \mu \mathrm{l}$ of saline. Experiments were performed 3 days after adenovirus injection unless otherwise indicated. Blood glucose and plasma insulin concentrations were determined with a GLUTEST PRO (Sanwa Kagaku Kenkyusho Co., Nagoya, Japan) and a mouse insulin ELISA kit (Seikagaku Co. Tokyo, Japan), respectively. For assaying time course of plasma insulin concentration in DNPI3K and LacZ mice, a Mouse Insulin ELISA KIT (Ultra-sensitivity) was used (Shibayagi Co., Shibukawa, Gunma, Japan). In those experiments, the plasma concentration of insulin in mice that had not been injected with adenovirus was below the limit of detection $(39 \mathrm{pg} / \mathrm{ml})$ of this ELISA kit. Other serum components were assayed with an autoanalyzer (model 7170; Hitachi High-Technologies Co., Tokyo, Japan). Statistical analysis was performed with Student $t$ tests.

Immunoblot analysis and assay of PI 3-K activity. Mice deprived of food for 16 hours were injected with human regular insulin (5 $\mathrm{U} / \mathrm{kg}$ of body mass) or saline via the tail vein. After 2 minutes, the animals were sacrificed, and the liver, hindlimb muscle, and epididymal fat were removed. Total homogenates were prepared from each tissue and subjected to immunoblot analysis
( $200 \mu \mathrm{g}$ of protein) or immunoprecipitation ( $\sim 5 \mathrm{mg}$ of protein). An $\mathrm{mAb}$ (G12) that recognizes bovine p85, but not rodent p85, was described previously (17). Polyclonal antibodies to p85, to IRS-1, to IRS-2, to phosphotyrosine (4G10), or to Akt1 were obtained from Upstate Biotechnology Inc. (Lake Placid, New York, USA); those to the $\beta$ subunit of the insulin receptor (IR) or to glycogen synthase kinase-3 $\beta$ (GSK3 $\beta$ ) were from Transduction Laboratories (Lexington, Kentucky, USA); and those specific for phosphorylated forms of Akt (phosphoSer ${ }^{473}$ ) or GSK3 (phospho-Ser ${ }^{9}$ ) were from Cell Signaling Technology (Beverly, Massachusetts, USA). PI 3-K activity in immunoprecipitates was assayed as described (3).

Oral glucose tolerance, insulin tolerance, and pyruvate challenge tests. For the oral glucose tolerance test, mice deprived of food for 16 hours were loaded orally with glucose $(2 \mathrm{~g} / \mathrm{kg})$ and killed 120 minutes later. The liver was removed and subjected to various assays. The glycogen (18) and glucose 6-phosphate (19) contents and glycogen synthase activity (20) were determined as described. For the insulin tolerance test, nonfasted mice were injected intraperitoneally with human regular insulin $(0.75 \mathrm{U} / \mathrm{kg})$. For the pyruvate challenge test, mice deprived of food for 16 hours were injected intraperitoneally with pyruvate dissolved in saline $(2 \mathrm{~g} / \mathrm{kg})$; where indicated, 3-mercaptopicolinic acid (3-MPA; $30 \mathrm{mg} / \mathrm{kg}$ ), an inhibitor of gluconeogenesis $(21,22)$, was administered orally 30 minutes before the test. The 3-MPA was synthesized as described (23).

Northern blot and real-time quantitative PCR analyses. For determination of the abundance of glucokinase, glucose6-phosphatase, phosphoenolpyruvate carboxykinase (PEPCK), and GLUT2 mRNAs, total RNA ( 15 $\mu \mathrm{g})$ extracted from the liver of mice that had been deprived of food for 16 hours was subjected to Northern blot analysis with ${ }^{32} \mathrm{P}$-labeled probes. Given that the hybridization signal intensity for sterol regulatory element-binding protein-1 (SREBP-1) mRNA from food-deprived mice was too weak for quantitative analysis, this mRNA was analyzed with total RNA prepared from nonfasted mice. Probes for glucokinase (24), SREBP-1 (25), PEPCK (26), and glucose-6-phosphatase (27) mRNAs were kindly provided by T. Noguchi (Nagoya University, Nagoya, Japan), H. Shimano (University of Tokyo), D.K. Granner (Vanderbilt University Medical Center, Nashville, Tennessee, USA), and H. Nakajima (Osaka Medical Center for Cancer and Cardiovascular Disease, Osaka, Japan), respectively, and that for rat GLUT2 mRNA was synthesized by PCR. Autoradiograms of Northern blots were visualized with a Fuji BAS2000 image analyzer (FujiFilm Co., Tokyo, Japan). For real-time quantitative PCR analysis, cDNA was synthesized from total RNA that had been extracted from the liver of mice deprived of food for 16 hours. The cDNA was then analyzed in a Sequence Detector (model 7900; PE Applied Biosystems) with specific primers and SYBR Green PCR Master (Perkin Elmer Life Sciences Inc., Boston, Massachusetts, USA). The relative abundance of mRNAs was calculated by the comparative cycle of threshold $\left(C_{\mathrm{T}}\right)$ method with 36B 4 mRNA as the 
a

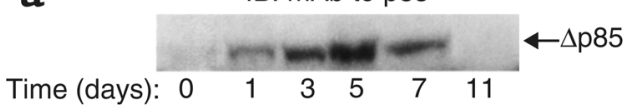

b

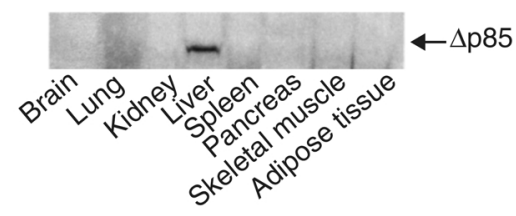

C

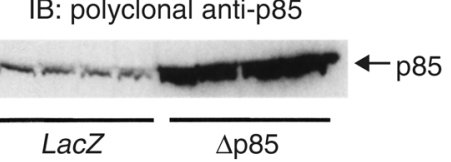

\section{Figure 1}

Expression of $\Delta p 85$ in the liver of mice injected with an adenoviral vector. (a) Time course of the expression of $\Delta p 85$ in the liver. The liver was removed from mice at the indicated times after injection with $A x C A \Delta p 85$, or from uninjected animals (day 0 ), and was subjected to immunoblot analysis (IB) with an $\mathrm{mAb}$ specific for bovine $\mathrm{p} 85$. (b) Total homogenates of the indicated tissues obtained from mice 3 days after injection with $\mathrm{AxCA} \Delta \mathrm{p} 85$ were subjected to immunoblot analysis with the $\mathrm{mAb}$ to bovine $\mathrm{p} 85$. (c) Liver homogenates prepared from mice 3 days after injection with $A x C A L a c Z$ or $A x C A \triangle p 85$ were subjected to immunoblot analysis with polyclonal antibodies that recognize both rodent and bovine $\mathrm{p} 85$. Data in $\mathbf{a}$ and $\mathbf{b}$ are representative of results obtained from three mice; those in c were obtained from four mice for each group.

invariant control. The primers used were as follows: IRS-1, 5'-GGCTGGCAATACGGT-3' (sense) and 5'GCCAAATCC-AGGTCTATGTA-3' (antisense); albumin, $5^{\prime}$ GACTTTGCACAGTTCCTGGATACA-3' (sense) and $5^{\prime}$ TTGTGGTTGTGATGTGTTTAGGCTA-3' (antisense); and 36B4, 5'-GGCCCTGCACTCTCGCTTTC-3' (sense) and 5'TGCC-AGGACG-CGCTTGT-3' (antisense).

\section{Results}

Expression of a dominant negative mutant of PI 3-K $(\Delta p 85)$ in mice by adenovirus-mediated gene transfer. Systemic infusion of adenoviral vectors has been shown to result in liver-specific expression of exogenous genes $(28,29)$. We therefore attempted to disrupt insulin-induced activation of PI 3-K in liver by an adenoviral vector (AxCA $\Delta \mathrm{p} 85$ ) encoding a dominant negative mutant of PI 3-K (3). This mutant protein, which corresponds to bovine $\mathrm{p} 85 \alpha$ without the binding site for the catalytic subunit, competes with endogenous regulatory subunits of class $\mathrm{I}_{A}$ PI 3-K in binding to tyrosine-phosphorylated proteins (17) and, consequently, prevents intracellular signaling transmitted through this lipid kinase (3-6). Mice were injected with $A x C A \Delta p 85$ via the tail vein, and expression of $\Delta p 85$ in liver was examined at various times thereafter by immunoblot analysis with an $\mathrm{mAb}$ that recognizes bovine, but not rodent, $\mathrm{p} 85$. The $\Delta \mathrm{p} 85$ protein was detected within 1 day of vector injection; its abundance was maximal at 3-5 days and decreased thereafter, returning to undetectable levels within 11 days (Figure 1a). Immunoblot analysis of various other tissues of the injected mice failed to detect $\Delta \mathrm{p} 85$ (Figure 1b). The amount of total (bovine and mouse) p85 protein in the liver of mice injected with AxCA $\triangle \mathrm{p} 85$ (DNPI3K mice) was at least five to six times that present in the liver of control mice injected with an adenoviral vector (AxCALac $Z$ ) encoding $\beta$-galactosidase (Figure 1c). The time course and tissue specificity of $\beta$-galactosidase expression in mice injected with AxCALacZ ( $L a c Z$ mice) were similar to those of $\Delta \mathrm{p} 85$ expression in DNPI3K mice (data not shown). We therefore performed experiments 3 days after adenovirus injection.

We next evaluated the effects of adenovirus infection on metabolic parameters. Mice were injected with $A x C A L a c Z$ or saline, and various metabolic parameters were measured at 3 days after the injection in the fasted state. There was no significant difference in plasma insulin and serum concentrations of triglycerides, cholesterol, and albumin (Table 1). Moreover, blood glucose concentrations in the fasted state and after oral glucose intake did not significantly differ between $\mathrm{Lac} Z$ mice and saline-injected mice. Although the serum levels of aspartate aminotransferase (AST) $(36 \pm 3.9 \mathrm{IU} / 1$ vs. $37.5 \pm 5.1 \mathrm{IU} / \mathrm{l})$ did not significantly differ between $L a c Z$ mice and saline-injected mice, those of alanine aminotransferase (ALT) were higher in LacZ mice than in saline-injected mice $(149 \pm 28$ vs. $82.2 \pm 7.3 \mathrm{IU} / \mathrm{l}$; $P<0.05$ ), probably due to the effect of adenovirus infection (data are means \pm SEM of values from four $L a c Z$ and five saline mice). We therefore performed most experiments with the use of $L a c Z$ mice as control hereafter. The serum levels of AST and ALT in DNPI3K mice $(221 \pm 33 \mathrm{IU} / 1$ and $80 \pm 2.0$, respectively) were higher than in LacZ mice (data are means \pm SEM of values from four mice; $P<0.01)$. Histological analysis revealed no signs of acute hepatic damage, such as infiltration of mononuclear cells, hepatocyte necrosis, or biliary congestion, in LacZ mice and DNPI3K mice (data not shown). The mass of the liver of DNPI3K

\section{Table 1}

Metabolic parameters in the fasted state and glucose concentrations during oral glucose tolerance test of $L a c Z$ mice and saline-injected mice 3 days after injection of AxCALacZ or saline

\begin{tabular}{cccc}
\hline & Parameter & LacZ & Saline \\
Fasted & Insulin $(\mathrm{ng} / \mathrm{ml})$ & $0.30 \pm 0.04$ & $0.41 \pm 0.11 \mathrm{NS}$ \\
Triglyceride $(\mathrm{mg} / \mathrm{dl})$ & $108.7 \pm 9.7$ & $117.6 \pm 12.8 \mathrm{NS}$ \\
Total cholesterol $(\mathrm{mg} / \mathrm{dl})$ & $104.2 \pm 4.1$ & $93.0 \pm 4.3 \mathrm{NS}$ \\
& Albumin $(\mathrm{g} / \mathrm{dl})$ & $3.02 \pm 0.06$ & $3.11 \pm 0.02 \mathrm{NS}$ \\
OGTT Glucose $(\mathrm{mg} / \mathrm{dl})$ & & \\
& $0 \mathrm{~min}$ & $58.2 \pm 3.5$ & $60.4 \pm 1.4 \mathrm{NS}$ \\
& $30 \mathrm{~min}$ & $298.6 \pm 15.9$ & $293.2 \pm 34.1 \mathrm{NS}$ \\
$60 \mathrm{~min}$ & $286.8 \pm 34.2$ & $323.6 \pm 23.1 \mathrm{NS}$ \\
& $120 \mathrm{~min}$ & $138.8 \pm 17.0$ & $159.0 \pm 14.3 \mathrm{NS}$
\end{tabular}

Data are means \pm SEM of values obtained from five mice. NS, not significant versus the corresponding value for $L a c Z$ mice. OGTT, oral glucose tolerance test. 
Table 2

Metabolic parameters of DNPI3K and LacZ mice measured in the fasted or nonfasted state 3 days after injection of $A x C A \Delta p 85$ or AxCALacZ

\begin{tabular}{cccc}
\hline & Parameter & LacZ & $\Delta \mathrm{p} 85$ \\
Fasted & Glucose $(\mathrm{mg} / \mathrm{dl})$ & $44.8 \pm 2.2$ & $52.4 \pm 2.6^{\mathrm{A}}$ \\
& Insulin $(\mathrm{ng} / \mathrm{ml})$ & $0.37 \pm 0.08$ & $1.50 \pm 0.18^{\mathrm{B}}$ \\
Triglyceride $(\mathrm{mg} / \mathrm{dl})$ & $83.5 \pm 13.1$ & $21.0 \pm 5.0^{\mathrm{A}}$ \\
FFA $(\mathrm{mEq} / \mathrm{l})$ & $2.05 \pm 0.21$ & $0.53 \pm 0.05^{\mathrm{B}}$ \\
Total cholesterol $(\mathrm{mg} / \mathrm{dl})$ & $136.7 \pm 5.3$ & $70.5 \pm 4.5^{\mathrm{B}}$ \\
Glycerol $(\mathrm{mg} / \mathrm{dl})$ & $6.50 \pm 0.75$ & $4.50 \pm 0.11^{\mathrm{A}}$ \\
Lactate $(\mathrm{mg} / \mathrm{dl})$ & $12.9 \pm 1.6$ & $9.0 \pm 1.2 \mathrm{NS}$ \\
Albumin $(\mathrm{g} / \mathrm{dl})$ & $3.00 \pm 0.04$ & $2.50 \pm 0.01^{\mathrm{B}}$ \\
Nonfasted & Glucose $(\mathrm{mg} / \mathrm{dl})$ & $110.5 \pm 5.8$ & $97.5 \pm 4.7 \mathrm{NS}$ \\
& Insulin $(\mathrm{ng} / \mathrm{ml})$ & $3.22 \pm 0.57$ & $5.86 \pm 0.41^{\mathrm{B}}$ \\
\hline
\end{tabular}

Data are means \pm SEM of values obtained from four to eight mice. ${ }^{A} P<0.05$, $\mathrm{B} P<0.01$; NS, not significant versus the corresponding value for $L a c Z$ mice.

mice was approximately $70 \%$ of that of $L a c Z$ mice $(3.55 \% \pm 0.09 \%$ vs. $5.06 \% \pm 0.10 \%$ body weight; means \pm SEM of values from six mice; $P<0.01)$.

Effects of $\Delta p 85$ on insulin-induced activation of signaling molecules and gene expression in liver. DNPI3K and LacZ mice were deprived of food for 16 hours and then injected with insulin or saline; then the liver was removed and subjected to various assays. Immunoprecipitation and immunoblot analysis revealed no difference in either the abundance of the IR or the extent of insulininduced tyrosine phosphorylation of the IR between the two groups of mice (Figure 2a). Unexpectedly, the abundance of IRS-1 was reduced by about $60 \%$ in DNPI3K mice; however, the extent of tyrosine phosphorylation of IRS-1 in the basal state was higher, and that in the insulin-stimulated state was only slightly lower, in DNPI3K mice than in LacZ mice. The amount of IRS-1 mRNA in the liver, as determined by real-time quantitative PCR analysis, did not differ between DNPI3K mice and LacZ mice $(0.091 \pm 0.008$ vs. $0.083 \pm 0.015 \mathrm{U}$, respectively, relative to the amount of $36 \mathrm{~B} 4$ mRNA; means \pm SEM of values from four mice), suggesting that the reduced abundance of IRS-1 protein in DNPI3K mice was attributable to a posttranscriptional event. Whereas the amount of IRS-2 did not differ between the two groups of mice, the extents of both basal and insulin-induced tyrosine phosphorylation of IRS-2 were increased in DNPI3K mice (Figure 2a). Although the mechanism responsible for the enhanced

\section{Figure 2}

Effects of $\Delta p 85$ on insulin-induced activation of signaling molecules and gene expression in the liver. (a) DNPI3K and LacZ mice were deprived of food for 16 hours and then injected with insulin $(+)$ or saline (-). The liver was removed 2 minutes later, homogenized, and subjected to immunoprecipitation (IP) with antibodies to the IR, to IRS-1, or to IRS-2. The resulting precipitates were subjected to immunoblot analysis with the same respective antibodies or with antibodies to phosphotyrosine (PY). Data are shown for two mice per condition and are representative of results obtained from six mice. (b) Immunoprecipitates prepared with antibodies to IRS- 1 or to IRS- 2 as in a were assayed for PI 3-K activity. Data are means \pm SEM of values from four mice. ${ }^{*} P<0.05$, ${ }^{*} P<0.01$ versus the corresponding value for LacZ mice. (c) Total RNA isolated from the liver of DNPI3K and of LacZ mice was subjected to Northern blot analysis with probes for the indicated mRNAs. Data are shown for three mice of each group. (d) Liver homogenates prepared as in a were subjected directly to immunoblot analysis with antibodies specific for phosphorylated Akt (pAkt) or for total Akt protein. Data are representative of results obtained from six mice. a
IP: anti-IR
IB: anti-PY
IP: anti-IR
IB: anti-IR

IP: anti-IRS-1

IB:anti-PY

IP: anti-IRS-1

IB: anti-IRS-1

IP: anti-IRS-2

IB: anti-PY

IP: anti-IRS-2

IB: anti-IRS-2

Insulin:

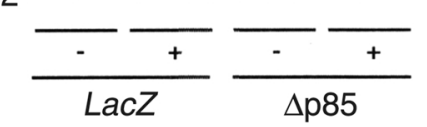

\section{C}
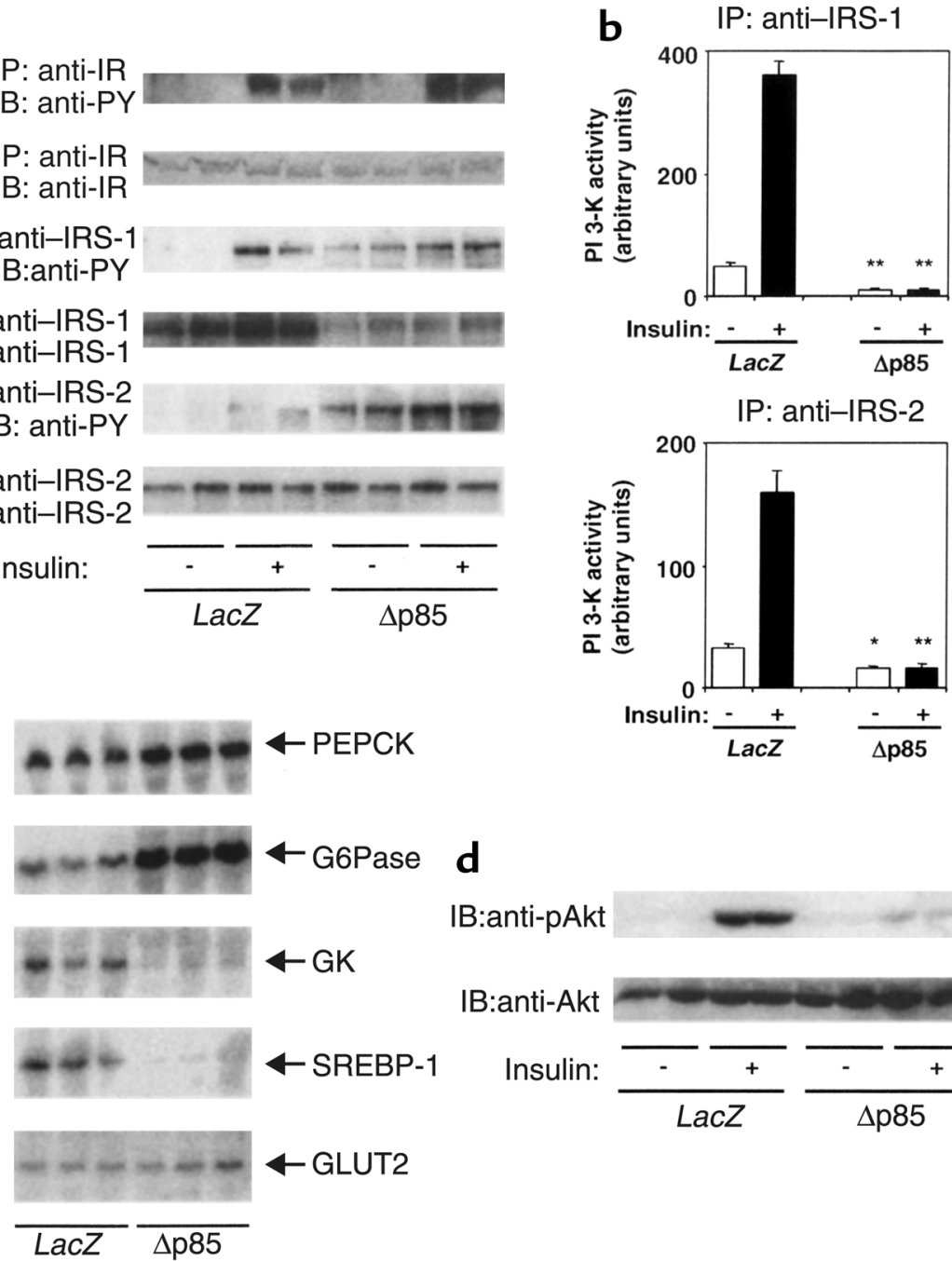

\section{d}

IB:anti-pAkt

IB:anti-Akt

Insulin:

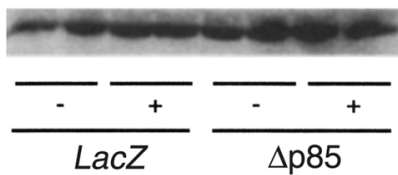


tyrosine phosphorylation of IRS proteins in DNPI3K mice is unknown, it is possible that prevention by $\Delta \mathrm{p} 85$ of negative feedback regulation of IRS phosphorylation may be a contributing factor.

The insulin-induced association of PI 3-K activity with either IRS- 1 or IRS- 2 was almost completely abolished in DNPI3K mice (Figure 2b), likely as a result of a dominant negative effect of $\Delta p 85$. Furthermore, the insulininduced phosphorylation of Akt, a protein kinase that functions downstream of PI 3-K $(1,2)$, was markedly inhibited in DNPI3K mice (Figure 2d); the abundance of Akt did not differ between DNPI3K and LacZ mice. These results thus indicate that almost complete inhibition of the insulin-induced activation of PI 3-K and of downstream effectors was achieved in the liver within 3 days after injection of $A x C A \Delta \mathrm{p} 85$.

Northern blot analysis of total RNA isolated from the liver of DNPI3K and $L a c Z$ mice revealed that the abundance of mRNAs for glucokinase and SREBP-1 was reduced whereas that of PEPCK and glucose-6-phosphatase mRNAs was increased in DNPI3K mice (Figure 2c). These data indicate that PI 3-K signaling contributes to the regulation of these genes in vivo, consistent with results obtained with cultured cells $(5,6,30,31)$. In contrast, the amount of GLUT2 mRNA did not differ between the two groups of mice.

Effects of $\Delta p 85$ on insulin-induced activation of signaling molecules in skeletal muscle and adipose tissue. Neither the abundance of the IR nor the extent of insulininduced tyrosine phosphorylation of the IR in skeletal muscle differed between DNPI3K and LacZ mice (Figure $3 a$ ). In addition, no marked differences were apparent in the amounts of IRS- 1 or IRS-2, in the extents of basal or insulin-induced tyrosine phosphorylation of IRS-1, or in the insulin-induced association of PI 3-K activity with IRS-1 or IRS-2 between skeletal muscle of DNPI3K mice and that of LacZ mice (Figure 3, a and b). The insulin-induced phosphorylation of Akt in skeletal muscle was also not impaired in DNPI3K mice (Figure 3c). Insulin signaling in skeletal muscle thus did not appear to be affected by expression of $\Delta \mathrm{p} 85$ in the liver.

The mass of epididymal adipose tissue in fasted DNPI3K mice was about 1.5 times that in fasted $L a c Z$ mice $(1.67 \% \pm 0.04 \%$ vs. $1.09 \% \pm 0.07 \%$ body weight, $P<0.01)$, whereas this parameter did not differ significantly between nonfasted DNPI3K and LacZ mice $(1.74 \% \pm 0.09 \%$ vs. $1.69 \% \pm 0.10 \%$ body weight, respectively; all data are means \pm SEM of values from six mice). Neither the abundance of the IR, IRS-1, or IRS-2 nor the extents of insulin-induced tyrosine phosphorylation of the IR or IRS- 1 in adipose tissue differed substantially between DNPI3K and LacZ mice (Figure 3a). The extent of insulininduced tyrosine phosphorylation of IRS-2 in both skeletal muscle and adipose tissue was too small for analysis (data not shown). The insulin-induced association of PI 3-K activity with IRS- 1 or IRS- 2 in adipose tissue was reduced by approximately $40 \%$ in DNPI3K mice compared with that apparent in LacZ mice (Figure 3b). The insulin-induced phosphorylation of Akt in adipose tissue, however, did not differ between the two groups of mice (Figure 3c). These results indicate that expression of $\Delta \mathrm{p} 85$ in the liver resulted in impairment of the activation of PI 3-K by insulin in adipose tissue and that the mass of this tissue in the fasted state was greater than that of control animals.

Effects of $\Delta p 85$ on metabolic parameters. There was no significant difference in body mass between DNPI3K and LacZ mice (22.8 \pm 0.5 vs. $21.8 \pm 0.4$ g, respectively; means \pm SEM of values from eight mice). The fasting blood a

b
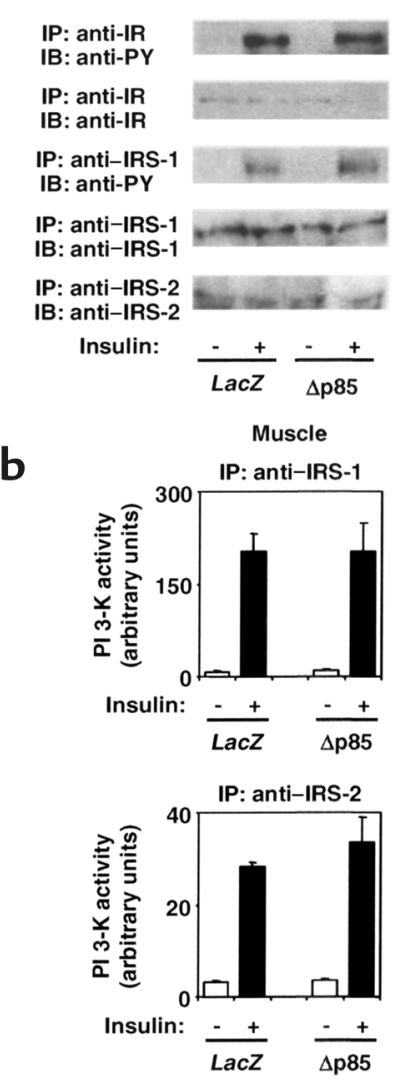

c

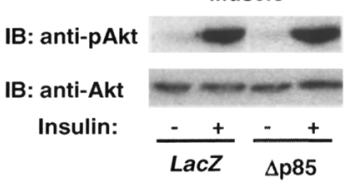

Adipose tissue

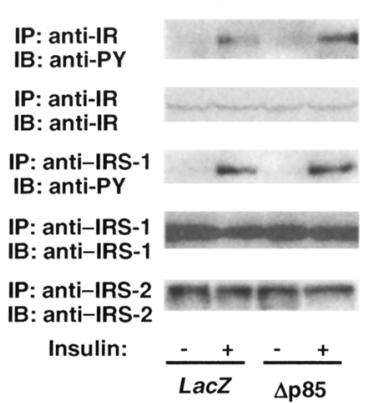

Adipose tissue

IP: anti-IRS-1
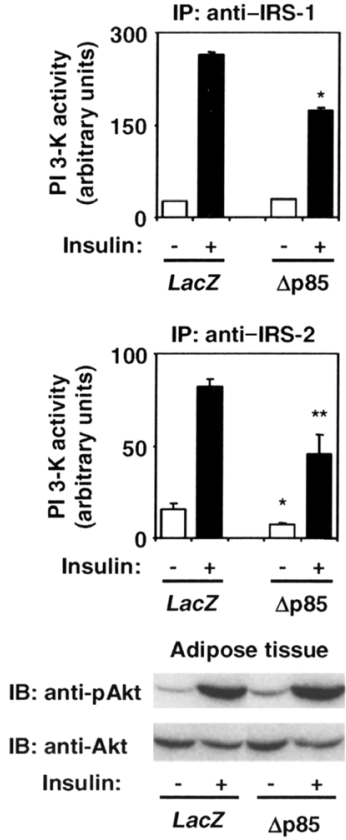

\section{Figure 3}

Effects of $\Delta p 85$ expression in the liver on insulin-induced activation of signaling molecules in skeletal muscle and adipose tissue. DNPI3K and LacZ mice were deprived of food for 16 hours and then injected with insulin $(+)$ or saline (-). Skeletal muscle (left panels) and adipose tissue (right panels) were removed 2 minutes later, homogenized, and subjected to analysis of the abundance and tyrosine phosphorylation of the IR, IRS-1, or IRS2 (a), of the association of PI 3-K activity with IRS-1 or IRS-2 (b), and of phosphorylation of Akt (c) as described in the legend to Figure 2. Data in $\mathbf{a}$ and $\mathbf{c}$ are representative of results obtained from three mice; those in $\mathbf{b}$ are means \pm SEM of values from four mice. ${ }^{*} P<0.05$, ${ }^{*} P<0.01$ versus the corresponding value for $L a c Z$ mice. 

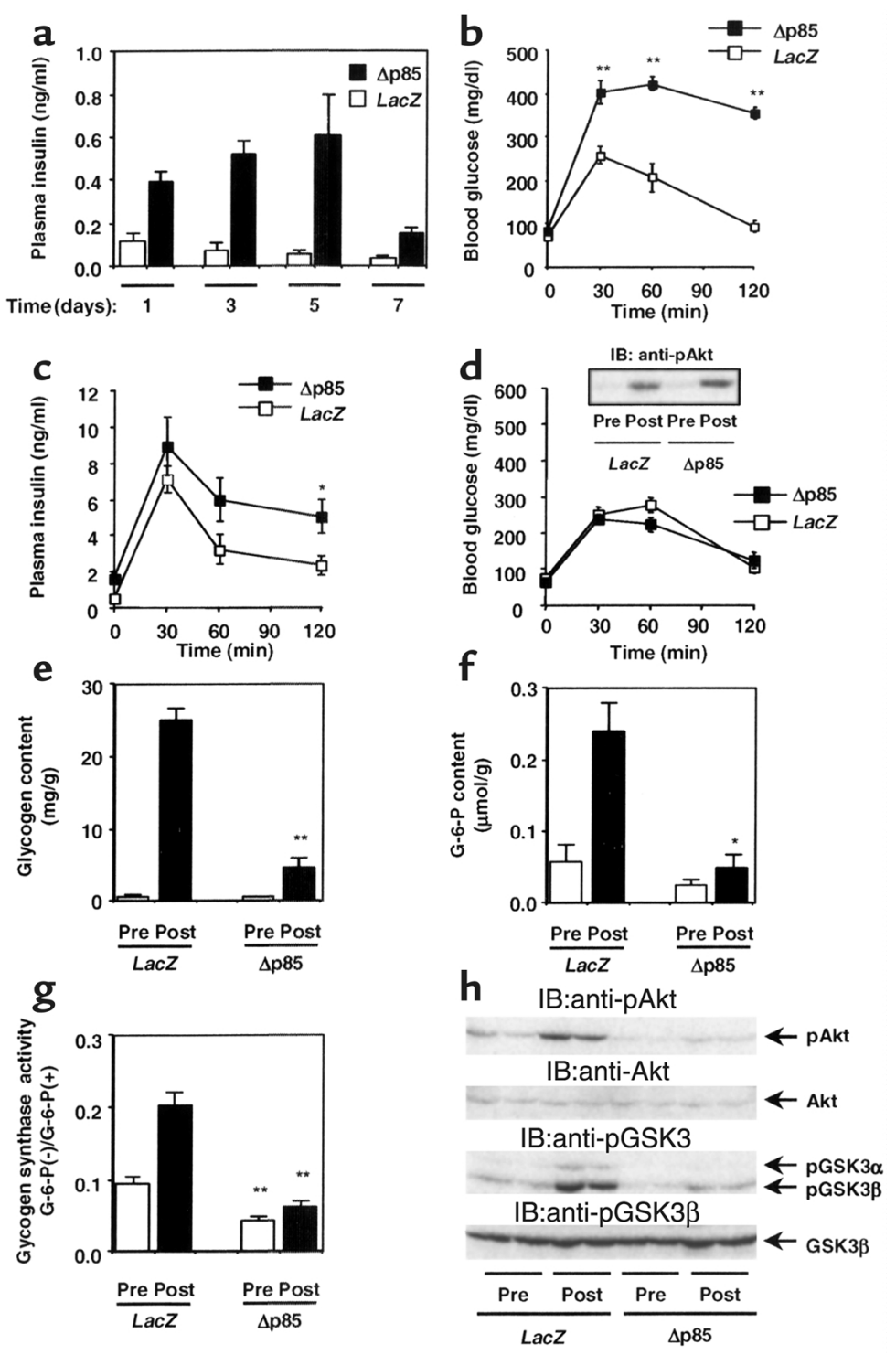

Figure 4

Time course of plasma insulin concentration in DNPI3K and LacZ mice, as well as the effects of $\Delta p 85$ expression in the liver on responses to oral glucose intake. (a) Plasma insulin concentration was measured at the indicated times after adenovirus injection and food deprivation for 16 hours. (b-g) DNPI3K and LacZ mice were deprived of food for 16 hours and then loaded orally with glucose $(2 \mathrm{~g} / \mathrm{kg})$ at $3(\mathbf{b}$, $\mathbf{c}$, and $\mathbf{e}-\mathbf{h}$ ) or 11 (d) days after the adenovirus injection. Blood glucose (b) and plasma insulin (c) concentrations were determined at the indicated times after glucose intake. Glycogen (e) and glucose 6-phosphate (f) contents, glycogen synthase activity ( $\mathbf{g})$, and phosphorylation of Akt (d, inset; and $\mathbf{h}$ ) and of GSK3 (h) in the liver were assayed before (Pre) and 120 minutes after (Post) glucose load. Glycogen and glucose 6-phosphate (G-6-P) contents are expressed per gram of liver mass. Glycogen synthase activity is expressed as the ratio of values determined in the absence of glucose 6-phosphate to values determined in the presence of glucose 6-phosphate. Data are means \pm SEM of values from three $(\mathbf{a})$, ten $(\mathbf{b})$, four $(\mathbf{c}, \mathbf{e}$ and $\mathbf{f})$, five $(\mathbf{d})$ or six (g) mice, or are shown for two mice and representative of six mice $(\mathbf{h})$ or shown for one mouse and representative of five mice ( $\mathbf{d}$, inset). ${ }^{*} P<0.05$, ${ }^{*} P<0.01 \mathrm{com}$ pared with the corresponding value for $L a c Z$ mice.

glucose concentration of DNPI3K mice was only slightly, but significantly, higher than that of LacZ mice (Table 2). In the nonfasted state, blood glucose concentrations were similar in both groups of mice. Plasma insulin concentrations of DNPI3K mice in the fasted and fed states were approximately 4 and 1.8 times, respectively, those of $L a c Z$ mice, suggesting that the inhibition of insulininduced activation of PI 3-K in the liver resulted in insulin resistance. The serum concentrations of FFAs, cholesterol, triglycerides, and glycerol (a precursor for gluconeogenesis) were significantly lower in DNPI3K mice than in LacZ mice. The serum concentration of lactate, another gluconeogenic substrate, was also lower in DNPI3K mice than in LacZ mice, but the difference was not statistically significant. The serum albumin concentration was reduced by approximately $17 \%$ in DNPI3K mice. The abundance of albumin mRNA in the liver was also reduced by more than $60 \%$ in DNPI3K mice compared with that in LacZ mice ( $42.8 \pm 8.4$ vs. $119.0 \pm 13.7 \mathrm{U}$ relative to the amount of $36 \mathrm{~B} 4 \mathrm{mRNA}$; means \pm SEM of values from four mice; $P<0.01$ ), consistent with the observation that the expression of albumin is regulated by insulin at the transcriptional level (32).

The plasma insulin concentration of DNPI3K mice was already higher than that of LacZ mice 1 day after vector injection, was maximal at 3-5 days, and had decreased markedly by 7 days (Figure 4a). The similarity in kinetics between the plasma insulin concentration and the expression of $\Delta \mathrm{p} 85$ in the liver suggests that the metabolic changes in DNPI3K mice result from inhibition of insulin-induced PI 3-K signaling in the liver.

Effects of $\Delta p 85$ on glucose and insulin tolerance. The blood glucose concentrations of DNPI3K mice were significantly greater than those of $L a C Z$ mice after oral glucose intake (Figure $4 \mathrm{~b}$ ). The marked hyperglycemia of DNPI3K mice persisted even 120 minutes after initiation of the test, when blood glucose concentration had returned to almost basal values in LacZ mice. Plasma insulin concentrations of DNPI3K mice were higher than those of $L a c Z$ mice at all times examined after oral glucose intake, although the difference was statistically significant only at 120 minutes (Figure 4c); these results are consistent with the notion that glucose intolerance in DNPI3K mice is attributable to insulin resistance. However, the glucose-lowering effect of exogenously administered insulin was similar in the two groups of mice (Figure 5a). At 11 days after the injection of the adenoviruses, at which time the abundance of $\Delta \mathrm{p} 85$ protein had returned to undetectable 
a

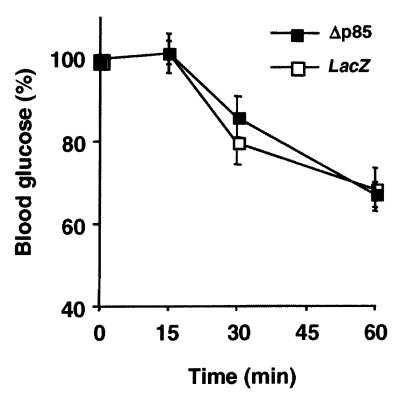

b

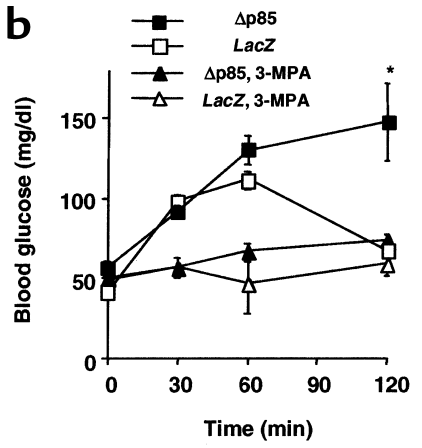

\section{Figure 5}

Effects of $\Delta p 85$ expression in the liver on insulin tolerance and pyruvate challenge. (a) DNPI3K and LacZ mice were injected intraperitoneally with insulin, and blood glucose concentration was measured at the indicated times thereafter. Data are expressed as a percentage of the initial glucose concentration and are means \pm SEM of values obtained from six mice. (b) DNPI3K and LacZ mice were deprived of food for 16 hours, orally administered with 3-MPA (or not), and, after $30 \mathrm{~min}$ utes, injected intraperitoneally with pyruvate. Blood glucose concentration was measured at the indicated times thereafter. Data are means \pm SEM of values obtained from four mice. ${ }^{*} P<0.05$ versus the corresponding value for $L a c Z$ mice. levels (Figure 1a), the blood glucose concentrations after oral glucose intake did not differ between DNPI3K and LacZ mice (Figure $4 \mathrm{~d}$ ).

Effects of $\Delta p 85$ on glycogen and glucose 6-phosphate accumulation during the glucose tolerance test. Hepatic glycogen content increased by a factor of about 40 within 120 minutes of oral glucose intake in $L a c Z$ mice, whereas this effect was reduced by more than $80 \%$ in DNPI3K mice (Figure 4e). The hepatic content of glucose 6-phosphate, an initial metabolite of glucose, in DNPI3K mice was also reduced by $60 \%$ and $80 \%$ before and after oral intake of glucose, respectively, compared with the corresponding values for $L a c Z$ mice (Figure $4 \mathrm{f}$ ).

Phosphorylation of Akt and that of GSK3, both of which are thought to contribute to the activation of glycogen synthase $(20,33)$, were stimulated in the liver of Lac $Z$ mice by glucose intake (Figure $4 \mathrm{~h}$ ). In contrast, the phosphorylation of both of these kinases in response to oral glucose intake was markedly impaired in the liver of DNPI3K mice. At 11 days after the adenovirus injection, phosphorylation of Akt induced by glucose intake did not differ between DNPI3K and $\mathrm{LacZ}$ mice (Figure $4 \mathrm{~d}$, inset). Oral glucose induced an approximately 2.5 -fold increase in the activity of glycogen synthase in the liver of $L a c Z$ mice (Figure $4 \mathrm{~g}$ ). The hepatic activity of glycogen synthase in DNPI3K mice before glucose intake was only about $50 \%$ of the corresponding value for $L a c Z$ mice, and the glucose-induced activation of this enzyme in the liver of DNPI3K mice was also markedly impaired.

Effect of $\Delta p 85$ on the pyruvate-induced increase in blood glucose concentration. Finally, we investigated the blood glucose response to administration of pyruvate, a precursor for gluconeogenesis. In $L a c Z$ mice, the blood glucose concentration was increased 30 minutes after pyruvate injection and had returned to almost basal levels within 120 minutes (Figure 5b). In DNPI3K mice, the increase in blood glucose concentration persisted at 120 minutes after pyruvate injection. The pyruvate-induced increase in blood glucose concentration was abolished by prior administration of 3-MPA, an inhibitor of gluconeogenesis $(21,22)$, in both groups of mice, suggesting that this effect of pyruvate was dependent on gluconeogenesis. These observations, together with the reduction in the serum concentrations of gluconeogenic precursors in
DNPI3K mice, suggest that a shortage of gluconeogenic precursors may prevent DNPI3K mice from increasing the fasting blood glucose level.

\section{Discussion}

Although in vitro studies have suggested that class $I_{A}$ PI 3-K plays an essential role in the metabolic actions of insulin $(1,2)$, targeted disruption of regulatory subunits of this enzyme resulted in apparently paradoxical phenotypes (11-14). We have now shown that systemic infusion of an adenoviral vector encoding a dominant negative mutant of PI 3-K $(\Delta \mathrm{p} 85)$ resulted in, within 3 days, liver-specific expression of the mutant protein and prevented both the insulin-induced association of PI 3-K with IRS proteins and the insulin-induced phosphorylation of Akt in liver. Selective expression of $\Delta \mathrm{p} 85$ in the liver also resulted in dyslipidemia and hyperinsulinemia and potentiated the increase in blood glucose concentration induced by oral glucose intake. These results provide direct evidence that the insulininduced activation of PI 3-K in the liver is required for metabolic actions of insulin in vivo.

An important characteristic of frank diabetes is fasting hyperglycemia, which is associated with an increase in hepatic glucose production (34). Although the insulin-induced activation of PI 3-K was almost completely abolished in the liver of DNPI3K mice, the fasting blood glucose concentration of these animals was increased only slightly. Similarly, in mice with liver-specific knockout of the IR gene (LIRKO mice), in which insulin action in the liver should be virtually abolished, basal hepatic glucose production was not increased and the fasting blood glucose concentration was only slightly increased, despite an enhanced expression of gluconeogenic enzymes in the liver (35). The abundance of gluconeogenic enzyme mRNAs in the liver was increased and the serum concentrations of gluconeogenic precursors were reduced in DNPI3K mice. In addition, the pyruvate-induced increase in blood glucose concentration, which was sensitive to a pharmacological inhibitor for gluconeogenesis, was enhanced in DNPI3K mice. These observations suggest that, in DNPI3K mice, and probably in LIRKO mice, a shortage of gluconeogenic substrates may prevent the development of marked fasting hyperglycemia. 
The exaggerated hyperglycemia apparent after oral glucose intake in DNPI3K mice might be attributable to a reduced ability of the liver to dispose of glucose as a result of multiple hepatic defects. Conversion of glucose to glucose 6-phosphate, catalyzed by gluco-kinase, is the initial step of glucose utilization, and the opposite reaction, catalyzed by glucose-6-phosphatase, is the final step of glucose production in the liver (36). The abundance of glucokinase mRNA was reduced and that of glucose-6-phosphatase mRNA was increased in the liver of DNPI3K mice. Changes in the amounts of these two key enzymes of glucose metabolism might thus account for a substantial proportion of the insufficiency of hepatic glucose disposal in DNPI3K mice. Indeed, the increase in hepatic glucose 6-phosphate content in response to oral glucose intake was markedly impaired in these animals. In addition, the activation of glycogen synthase, as well as the phosphorylation of Akt and of GSK3 in the liver, induced by oral glucose intake was also greatly attenuated in DNPI3K mice. Suppression of glycogen synthase activity and the reduced ability to accumulate glucose 6-phosphate likely contribute to the impaired hepatic glycogen storage in these animals. Glucose 6-phosphate is a potent allosteric activator of glycogen synthase (36). The reduction in glycogen synthase activity in DNPI3K mice might thus be attributable both to direct inhibition of the insulin-induced activation of PI 3-K and the consequent prevention of Akt-GSK3 signaling, and to the reduction in hepatic glucose 6-phosphate content.

Despite the pronounced hyperglycemia observed in response to oral glucose intake in DNPI3K mice, the blood glucose concentration of these animals in the random-fed state was not significantly increased. This observation suggests that, whereas normal hepatic PI 3-K signaling is essential for disposal of an acutely administered energy source, glucose homeostasis in the random-fed state was adequately compensated in DNPI3K mice. Mice eat smaller quantities of food but eat more frequently over a longer portion of the day than do humans. Our data are thus consistent with the improvement of glycemic control apparent in diabetic individuals taking smaller but more frequent meals (37).

Inhibition of hepatic insulin signaling for a period as short as 3 days still influenced the function of other organs in DNPI3K mice. In the fasted state, the mass of adipose tissue of DNPI3K mice was about 1.5 times that of LacZ mice; this is likely attributable to reduced lipolysis as a result of hyperinsulinemia. Consistent with this notion, the serum concentrations of FFAs and glycerol, both of which are produced by hydrolysis of triglycerides, were reduced in DNPI3K mice. Whereas the insulin-induced association of PI 3-K activity with IRS-1 or IRS-2 in muscle was not affected by expression of $\Delta \mathrm{p} 85$ in the liver, these effects of insulin were reduced by approximately $40 \%$ in adipose tissue. It is possible that the hyperinsulinemia or the greater adipose tissue mass apparent in DNPI3K mice was responsible for these defects in insulin signaling. Despite the reduced extent of insulin-induced PI 3-K activation, the phosphorylation of Akt in response to insulin was not affected in adipose tissue of DNPI3K mice. A similar discrepancy between PI 3-K activity and Akt phosphorylation has been described in humans with type 2 diabetes (8). These observations might be explained if maximal stimulation of PI 3-K is not necessary for maximal phosphorylation of Akt in vivo.

Insulin stimulates the production of triglycerides by upregulating the expression of lipogenic enzymes in the liver in an SREBP-1-dependent manner $(25,30)$. It also promotes the clearance of serum triglycerides by stimulating the expression and secretion of lipoprotein lipase in adipose tissue (38). The reduced concentration of triglycerides in the serum of DNPI3K mice may thus be related to the impairment of insulin action in the liver as well as to an enhanced action of insulin in peripheral tissues.

The metabolic characteristics of DNPI3K mice resemble those of LIRKO mice in several respects (35). Both animal models exhibit hyperinsulinemia, an exaggerated increase in blood glucose concentration in response to glucose challenge, and a relatively minor alteration in fasting blood glucose concentration. Moreover, the features of dyslipidemia and the changes in the amounts of mRNAs for various hepatic enzymes are similar in the two models. However, the metabolic abnormalities of LIRKO mice are more pronounced than are those of DNPI3K mice: The plasma insulin concentration is much higher in the former animals, which, unlike DNPI3K mice, also exhibit hyperglycemia in the nonfasted state (35).

It is possible that the more pronounced metabolic alterations in LIRKO mice are due not only to the direct inhibition of hepatic insulin action but also to secondary effects of severe long-term hyperinsulinemia. Indeed, the glucose infusion rate during a hyperinsulinemic-euglycemic clamp study, which reflects peripheral (but not hepatic) insulin sensitivity (34), was shown to be reduced for LIRKO mice, probably as a result of the chronic hyperinsulinemia (39). In addition, we have found that expression from an adenoviral vector (AxCADNIR) of a dominant negative mutant of the IR in liver, which resulted in greater-than- $90 \%$ inhibition of the insulininduced increase in PI 3-K activity in this organ, led to hyperinsulinemia and glucose intolerance similar to those apparent in DNPI3K mice (K. Miyake et al., unpublished observations). In LIRKO mice (35), but not in DNPI3K mice or in AxCADNIR-injected mice (K. Miyake et al., unpublished observations), the acute glucose-lowering effect of exogenous insulin was severely impaired. These differences also may be explained by the peripheral insulin resistance apparent in LIRKO mice. The abundance of IRS-1, but not that of IRS-2, was reduced in the liver of DNPI3K mice. Although the mechanism of this effect is not clear, it is likely mediated at the posttranscriptional level, given that the amount of IRS-1 mRNA was not reduced. We cannot exclude the possibility that inhibition of IRS-1 signaling pathways other than 
that mediated by PI3-K contributes to the metabolic features of DNPI3K mice. However, heterozygous knockout of the IRS-1 gene, which resulted in an approximately $50 \%$ decrease in the abundance of IRS- 1 protein throughout the body, did not induce marked changes in metabolic parameters $(40,41)$, suggesting that the metabolic characteristics of DNPI3K mice are dependent on the inhibition of PI 3-K signaling, not on suppression of other signaling pathways mediated by IRS- 1 .

In summary, liver-specific expression of a dominant negative mutant of class $\mathrm{I}_{A}$ PI 3-K resulted in insulin resistance, exaggerated hyperglycemia in response to oral glucose intake, and a reduced capacity of the liver to accumulate glucose 6-phosphate and glycogen. The mass of adipose tissue of DNPI3K mice in the fasted state was greater than that of control animals. The DNPI3K animals also exhibited impaired activation of PI 3-K in adipose tissue, probably as a result of the hyperinsulinemia induced by inhibition of insulin signaling in the liver. We believe our results provide the first evidence that PI 3-K is essential for metabolic regulation by insulin in living animals.

\section{Acknowledgments}

We thank I. Saito, T. Noguchi, H. Shimano, H. Nakajima, and D.K. Granner for materials, T. Ohara for helpful discussions, and $\mathrm{H}$. Iguci for quantitative PCR analysis. This work was supported by a grant from the Ministry of Education, Culture, Sports, Science, and Technology of Japan, a grant from Naito Foundation (to W. Ogawa), and a grant-in-aid for the Research for the Future Program from the Japan Society for the Promotion of Science (to M. Kasuga).

1. Ogawa, W., Matozaki, T., and Kasuga, M. 1998. Role of binding proteins to IRS-1 in insulin signalling. Mol. Cell. Biochem. 182:13-22.

2. Shepherd, P.R., Withers, D.J., and Siddle, K. 1998. Phosphoinositide 3kinase: the key switch mechanism in insulin signalling. Biochem. J. 333:471-490.

3. Sakaue, H., et al. 1997. Phosphoinositide 3-kinase is required for insulin-induced but not for growth hormone- or hyperosmolarityinduced glucose uptake in 3T3-L1 adipocytes. Mol. Endocrinol. 11:1552-1562

4. Kitamura, T., et al. 1999. Insulin-induced phosphorylation and activation of cyclic nucleotide phosphodiesterase $3 \mathrm{~B}$ by the serine-threonine kinase Akt. Mol. Cell. Biol. 19:6286-6296.

5. Kotani, K., et al. 1999. Dominant negative forms of Akt (protein kinase B) and atypical protein kinase $\mathrm{C} \lambda$ do not prevent insulin inhibition of phosphoenolpyruvate carboxykinase gene transcription. J. Biol. Chem. 274:21305-21312.

6. Matsumoto, M., et al. 2002. Role of the IRS-1 and PI 3-kinase signaling pathway in insulin-induced expression of SREBP-1c and glucokinase genes in rat hepatocytes. Diabetes. 51:1672-1680.

7. Heydrick, S.J., et al. 1993. Defect in skeletal muscle phosphatidylinositol3-kinase in obese insulin-resistant mice. J. Clin. Invest. 91:1358-1366.

8. Kim, Y.B., Nikoulina, S.E., Ciaraldi, T.P., Henry, R.R., and Kahn, B.B. 1999. Normal insulin-dependent activation of Akt/protein kinase B, with diminished activation of phosphoinositide 3-kinase, in muscle in type 2 diabetes. J. Clin. Invest. 104:733-741.

9. Tsuchida, A., et al. 2001. The effects of brain-derived neurotrophic factor on insulin signal transduction in the liver of diabetic mice. Diabetologia. 44:555-566.

10. Bi, L., Okabe, I., Bernard, D.J., Wynshaw-Boris, A., and Nussbaum, R.L. 1999. Proliferative defect and embryonic lethality in mice homozygous for a deletion in the $\mathrm{p} 110 \alpha$ subunit of phosphoinositide 3-kinase. J. Biol. Chem. 274:10963-10968.

11. Fruman, D.A., et al. 2000. Hypoglycaemia, liver necrosis and perinatal death in mice lacking all isoforms of phosphoinositide 3-kinase p $85 \alpha$. Nat. Genet. 26:379-382.
12. Mauvais-Jarvis, F., et al. 2002. Reduced expression of the murine p $85 \alpha$ subunit of phosphoinositide 3 -kinase improves insulin signaling and ameliorates diabetes. J. Clin. Invest. 109:141-149. doi:10.1172/JCI200213305.

13. Ueki, K., et al. 2002. Increased insulin sensitivity in mice lacking p85 $\beta$ subunit of phosphoinositide 3-kinase. Proc. Natl. Acad. Sci. USA. 99:419-424.

14. Terauchi, Y., et al. 1999. Increased insulin sensitivity and hypoglycaemia in mice lacking the p $85 \alpha$ subunit of phosphoinositide 3-kinase. Nat. Genet. 21:230-235.

15. Abel, E.D., et al. 2001. Adipose-selective targeting of the GLUT4 gene impairs insulin action in muscle and liver. Nature. 409:729-733.

16. Kim, J.K., et al. 2001. Glucose toxicity and the development of diabetes in mice with muscle-specific inactivation of GLUT4. J. Clin. Invest. 108:153-160. doi:10.1172/JCI200110294.

17. Hara, K., et al. 1994. 1-Phosphatidylinositol 3-kinase activity is required for insulin-stimulated glucose transport but not for RAS activation in CHO cells. Proc. Natl. Acad. Sci. USA. 91:7415-7419.

18. Hassid, W.Z., and Abraham, S. 1957. Chemical procedures for analysis of polysaccharide. Methods Enzymol. 3:34-36.

19. Hohorst, H.J. 1965. D-Glucose-6-phosphate and D-fructose-6-phosphate. In Methods of enzymatic analysis. H.U. Bergmeyer, editor. Academic Press. New York, New York, USA. 134-138.

20. Takata, M., et al. 1999. Requirement for Akt (protein kinase B) in insulininduced activation of glycogen synthase and phosphorylation of 4E-BP1 (PHAS-1). J. Biol. Chem. 274:20611-20618.

21. DiTullio, N.W., et al. 1974. 3-Mercaptopicolinic acid, an inhibitor of gluconeogenesis. Biochem. J. 138:387-394.

22. Podolin, D.A., Pagliassotti, M.J., Gleeson, T.T., and Mazzeo, R.S. 1994. Influence of endurance training on the age-related decline in hepatic glyconeogenesis. Mech. Ageing Dev. 75:81-93.

23. Memoli, K.A. 1996. A convenient preparation of 3-mercaptopicolinic acid. Tetrahedron Lett. 37:3617-3618.

24. Matsuda, T., Noguchi, T., Yamada, K., Takenaka, M., and Tanaka, T. 1990. Regulation of the gene expression of glucokinase and L-type pyruvate kinase in primary cultures of rat hepatocytes by hormones and carbohydrates. J. Biochem. (Tokyo). 108:778-784.

25. Shimano, H., et al. 1996. Overproduction of cholesterol and fatty acids causes massive liver enlargement in transgenic mice expressing truncated SREBP-1a. J. Clin. Invest. 98:1575-1584.

26. Beale, E.G., et al. 1985. Rat hepatic cytosolic phosphoenolpyruvate carboxykinase (GTP). Structures of the protein, messenger RNA, and gene. J. Biol. Chem. 260:10748-10760.

27. Shingu, R., et al. 1996. Expression and distribution of glucose-6-phosphatase catalytic subunit messenger RNA and its changes in the diabetic state. Res. Commun. Mol. Pathol. Pharmacol. 93:13-24.

28. Jaffe, H.A., et al. 1992. Adenovirus-mediated in vivo gene transfer and expression in normal rat liver. Nat. Genet. 1:372-378.

29. Trinh, K.Y., O'Doherty, R.M., Anderson, P., Lange, A.J., and Newgard, C.B. 1998. Perturbation of fuel homeostasis caused by overexpression of the glucose-6-phosphatase catalytic subunit in liver of normal rats. J. Biol. Chem. 273:31615-31620.

30. Azzout-Marniche, D., et al. 2000. Insulin effects on sterol regulatory-element-binding protein-1c (SREBP-1c) transcriptional activity in rat hepatocytes. Biochem. J. 350:389-393.

31. Dickens, M., Svitek, C.A., Culbert, A.A., O’Brien, R.M., and Tavare, J. M. 1998. Central role for phosphatidylinositide 3-kinase in the repression of glucose6-phosphatase gene transcription by insulin. J. Biol. Chem. 273:20144-20149.

32. Flaim, K.E., et al. 1985. Direct effect of insulin on albumin gene expression in primary cultures of rat hepatocytes. Am. J. Physiol. 249:E447-E453.

33. Cross, D.A., Alessi, D.R., Cohen, P., Andjelkovich, M., and Hemmings, B.A. 1995. Inhibition of glycogen synthase kinase- 3 by insulin mediated by protein kinase B. Nature. 378:785-789.

34. DeFronzo, R.A. 1997. Pathogenesis of type 2 diabetes: metabolic and molecular implications for identifying diabetic genes. Diabetes Rev. 5:177-269.

35. Michael, M.D., et al. 2000. Loss of insulin signaling in hepatocytes leads to severe insulin resistance and progressive hepatic dysfunction. Mol. Cell. 6:87-97.

36. Radziuk, J., and Pye, S. 2001. Hepatic glucose uptake, gluconeogenesis and the regulation of glycogen synthesis. Diabet. Metab. Res. Rev. 17:250-272.

37. Jenkins, D.J. 1997. Carbohydrate tolerance and food frequency. Br. J. Nutr. 77(Suppl. 1):S71-S81.

38. Oliver, J.D., and Rogers, M.P. 1993. Stimulation of lipoprotein lipase synthesis by refeeding, insulin and dexamethasone. Biochem. J. 292:525-530.

39. Fisher, S.J., and Kahn, C.R. 2001. No evidence for an indirect effect of insulin to suppress glucose production in liver-specific insulin receptor knockout mice. Diabetes. 50(Suppl. 2):A50. (Abstr.)

40. Tamemoto, H., et al. 1994. Insulin resistance and growth retardation in mice lacking insulin receptor substrate-1. Nature. 372:182-186.

41. Araki, E., et al. 1994. Alternative pathway of insulin signalling in mice with targeted disruption of the IRS-1 gene. Nature. 372:186-190. 